\title{
Real Time Driver Fatigue Recognition Based on Image Processing
}

\author{
Bhuvaneshwari Ingale ${ }^{1}$, Kanchan Wasnik ${ }^{2}$, Megha Peddawad ${ }^{3}$, Kavita Deore ${ }^{4}$ \\ Indira College of Engineering and Management, Maval, Pune ${ }^{1}$
}

\begin{abstract}
Driver fatigue is the main reason of serious damages among all other road accidents. Thus, a new system is proposed with a modern approach which will detect driver fatigue by considering most of the fatigue symptoms such as eye closure, yawning, head tilting. Inattentive vehicle movement on the road under fatigue condition is also considerable for driver fatigue. The approach of this system is to detect these symptoms for the best driving condition on the road. These symptoms are monitored by using two cameras. Thus, a robust system is proposed where head tilting, facial expressions and lane departure for fatigue will be detected collectively. First step of this system is to visualize the face of the driver from one camera while another camera is used to track the road. Then face, eye and mouth (check for yawning) detections of the driver are performed. Facial detection of our method is free from any kind of wrong or bogus detection. Besides, the detection process can strongly determine the desired part of the face in spite of poor light intensity of camera and more realistic to implement. Head tilting detection makes this approach more promising. Lane departure detection in the approach gives this system an advanced level. Lane detection measures the distance of the vehicle between two lanes. Alarm system has been made which will be activated if any fault is found through these detection procedures.
\end{abstract}

Keywords: Driver fatigue, Fatigue detection, Fatigue monitoring, Head tilting, Lane departure, OpenCV.

\section{INTRODUCTION}

Nowadays most of the road accidents are caused due to driver fatigue near about $20 \%$ of accidents are caused due to fatigue. Fatigue is the condition which is occurred because of overstress. Proposed system is made to reduce the accidents by monitoring the activities of driver. Approach of this system is to detect the driver fatigue by using nonintrusive technique. Nonintrusive technique used to measure visual behaviors such as eye conditions (open or close), head tilting, yawning. Another one method is driver performance analysis which is used for monitoring the vehicles on road and lane detection. Non-intrusive methods are helpful to detect facial expressions for fatigue recognition Eye closure and mouth gaping are popular symptoms in the driver fatigue recognition [1]. This is real time system which will consider all fatigue symptoms collectively such as eye closure, head tilting (where head is moving to the left or right) yawning, lanedetection. This system uses OpenCV (Open Source Computer Vision) libraries for implementation. There are two cameras used in this system first camera is used to keep watch on face of the driver and another i.e,2nd camera is used to keep watch on the road for lane detection.First camera detects the yawning,eye closure or open,head tilting. This first camera is mounted on the steering wheel and it continuously detectthe face of the driver.Face detection in this system is free from any wrong or bogus detection because it is real time system second camera is used for the lane detection method and it measures the distance of the vehicle from both two lanes. Alarm system has been made which will be activated if any fault is found through these detection procedures.

\section{LITERATURE SURVEY}

There have been vast researches for fatigue recognition using different techniques. Physiological measurement is prevailing on the research because of its accuracy. The work in paper A neural-network-based system for monitoring driver fatigue, by S. Ribaric, J. Lovrenci and N. Pavesi, described neural network based system to detect face, mouth and head position. The warningor alarmsystem is also described to raise drive alertness. In paper Detecting Driver Yawning in Successive Images L. Yufeng and W.Zengcai proposed that face region detection can be attained using the difference between two images. The distance between the midpoint of nostrilsand chin is then used to detect yawn of the driver[2].The work presented in Real time and non-intrusive driver fatigue monitoring, by J. Jimenez-Pinto, M. Torres-Torrit suggested that fatigue can be identifiedconsidering the drivers facial pose. Head tilts can be detected by analyzing facial orientation. All the first generations of lane detection systems wereedge-based. They relied on thresholding the image intensity to detectpotential lane edges, followed by a perceptual grouping of the edge points to detect the lane markers of interest [3].

The work in Real-Time Lane Departure Detection Based on Extended Edge-Linking Algorithm proposed that region of interest (ROI) can be extracted for lane departure. Hough transform is used to detect the lines of the road in ROI. A 
lane departure decision is made using the distance of Hough origin and lane-line midpoint.

In paper "A Driver Warning System Based on the LOIS Lane Detection Algorithm" Chriskreucher, Karl kluge used the LOIS (Likelihood of Image Shape) algorithm. This algorithm finds the lane markers in robust way even in the presence of shadowing, varied lighting conditions. LOIS algorithm checks for the sequence of images after video acquisition, and raised the alarm for warning if a lane crossing is impending [4].

\section{III.PROPOSED SYSTEM}

In this system integration of all modules is done to detect the driver fatigue. Proposed system is implemented in such way that if any caused is found through this detection procedure then alarm is raised to alert the driver.

\section{A. Face Detection}

Face detection is the first stage. There are numerous challenges that are belongs to the face detection such as position, orientation, lighting conditions, variability of size, shape and color [1].

Generally, camera is fixed at the front of the driver so it is easy for detection of face and head tilt determination. For solving the problem of brightness skin color segmentation is used which converts the RGB to YCbCr.Erosion and dilation are also used. Erosion removes the noise and dialation is used for enhancing the strength of skin color.

\section{B. Eye Detection}

When eyes are closed for the certain period of time then it is called as one of the driver fatigue condition. It is the second stage for detecting driver fatigue condition. After that video is acquired of face images and converted into number of frames and eye region is extracted from face region. If the eyes are closed in alternate frames then we can conclude that it is "normal eye blinking", If the eyes are closed in continuous frames then we can conclude that driver is in fatigue condition.

\section{Mouth Detection}

Mouth detection is one of the factor for driver fatigue detection. mouth detection is the requirement for yawn detection. Yawn is detected by extracting the region of interest of mouth from face region. Yawn is detected by finding the difference between features of normal mouth and yawning mouth. Here erosion and dialation are used for finding the Region of Interest of mouth.

\section{Yawn Detection}

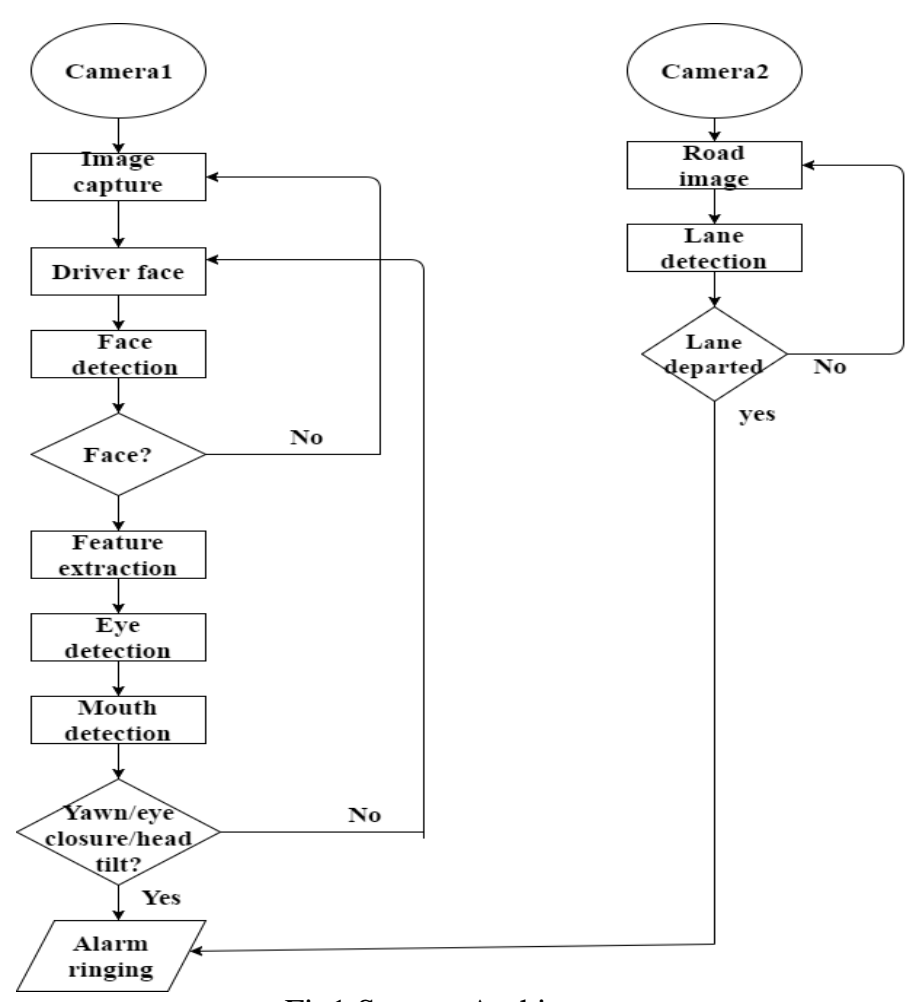

Fig1.System Architecture 
In yawn detection, Non-skin color segmentation is used for detecting yawning. The ROI is used for Mouth detection. Yawning mouth is different than the normal mouth because in case of yawning largest hole is detected in face and eye area becomes narrow at the time of yawning. So, it is helpful for detecting the yawn by considering the largest hole in the face as yawn.

\section{IV.CONCLUSION}

A real-time approach has been proposed for detection of driver fatigue recognition in such a way that it will consider most of the fatigue symptoms. This system has become robust because of lane detection. Less algorithms are used for eye, mouth and yawn detection because of OpenCV (Open source computer vision) library.

\section{REFERENCES}

[1] Rafi Ahmed, Kazi Emrul Kayes Emon, Md. Foisal Hossain," Robust Driver Fatigue RecognitionUsing Image Processing”,3rd InternationalConferenceon Informatics, Electronics \& Vision,2014.

[2] L. Yufeng, W. Zengcai, "Detecting Driver Yawning in Successive Images",1st International Conferenceon Bioinformatics and Biomedical Engineering, pp, 2007.

[3] J. Jimenez-Pinto, M. Torres-Torriti," Driver alert state and fatigue detection by salient points analysis", IEEE International Conference on Systems, Man and Cybernetics,2009.

[4] Chris Kreucher, Sridhar Lakshmanan, and Karl Kluge," A Driver Warning System Based on the LOIS Lane Detection Algorithm" University of Michigan-Dearborn Vehicle Electronics Lab 4901 Evergreen Road, Dearborn, MI 48128-1491 USA. 